\title{
ALGEBRAIC POINTS ON QUARTIC CURVES OVER FUNCTION FIELDS
}

\author{
by. ANDREW BREMNER
}

(Recieved 4 October, 1984)

1. The following general problem is of interest. Let $\Gamma$ be an irreducible algebraic variety of degree $d$, in projective $n$-space $\mathbf{P}^{n}$, defined over a field $k$; and suppose that $K$ is a finite extension of $k$ with [ $K: k]$ prime to $d$. If $\Gamma$ has a point defined over $K$, then does it necessarily have a point defined over $k$ ?

It has been studied in various instances by several authors: see, for example, Cassels [2], Coray [3, 4], Pfister [5], Bremner, Lewis, Morton [1]. Coray [3] shows that a quartic curve $\Gamma$ over $\mathbf{Q}$ may possess points in extension fields of $\mathbf{Q}$ of every odd degree greater than one, but have no points in $\mathbf{Q}$ itself. Some further examples of this instance occur in the paper of Bremner, Lewis, Morton, with the additional property that the curve $\Gamma$ also possesses points in every $p$-adic completion $\mathbf{Q}_{p}$ of $\mathbf{Q}$.

When the ground field is the function field $k=\mathbf{Q}(\lambda)$ of transcendence degree 1 , then Bremner, Lewis, Morton again give (although rather as a rabbit from a hat) two examples to show that a quartic curve $\Gamma$ defined over $k$ may possess points in extension fields of $k$ of every odd degree greater than one, but have no points in $k$ itself. It is the purpose of this note to give a method whereby an infinite family of such curves $\Gamma$ may be produced (of which the two examples of Bremner, Lewis, Morton are special cases).

2. From Coray [3] it follows that if the quartic curve $\Gamma$ (of genus 3, when irreducible) possesses a point in a cubic extension of $k$, then it will possess points in extension fields of $k$ of every odd degree greater than one. So it will suffice to produce a family of curves having points in a cubic extension of $k$. We search for polynomial identities of the following type:

$$
\left(x^{3}+a x^{2}+b x+c\right)\left(x^{3}-a x^{2}+b x-c\right)\left(x^{2}+d\right)=\left(x^{2}+e\right)^{4}+M x^{4}+N .
$$

Such an identity implies that the diagonal form $X^{4}+M Y^{4}+N Z^{4}=0$ representing a quartic curve does indeed have zeros in the field defined by a root of the cubic polynomial $x^{3}+a x^{2}+b x+c$. It will then remain to ensure that this cubic is irreducible, and that there are no global points on the quartic curve.

Equating coefficients at (1) gives

$$
\begin{aligned}
d+2 b-a^{2} & =4 e \\
b^{2}-2 a c+\left(2 b-a^{2}\right) d & =6 e^{2}+M \\
d\left(b^{2}-2 a c\right)-c^{2} & =4 e^{3} \\
-d c^{2} & =e^{4}+N
\end{aligned}
$$

Glasgow Math. J. 26 (1985) 187-190. 
and eliminating $d$ between (2), (4) gives

$$
c^{2}+2 c\left(a^{3}-2 a b+4 a e\right)+4 e^{3}-4 e b^{2}+2 b^{3}-a^{2} b^{2}=0 .
$$

Thus

where

$$
c=-a^{3}+2 a b-4 a e+\Delta
$$

$$
\Delta^{2}=a^{6}-4 a^{4}(b-2 e)+a^{2}\left(5 b^{2}-16 b e+16 e^{2}\right)+\left(-2 b^{3}+4 b e^{2}-4 e^{3}\right) .
$$

Put now

and

$$
\lambda=b / e, \quad p(\lambda)=\lambda^{3}-2 \lambda^{2}+2
$$

$$
\rho=-2 p(\lambda) e / a^{2}, \quad \sigma=2 p(\lambda) \Delta / a^{3} .
$$

Then (7) becomes

$$
\sigma^{2}=\rho^{3}+\left(5 \lambda^{2}-16 \lambda+16\right) \rho^{2}+8(\lambda-2)\left(\lambda^{3}-2 \lambda^{2}+2\right) \rho+4\left(\lambda^{3}-2 \lambda^{2}+2\right)^{2}
$$

and from (2)-(6), (8), (8)

$$
\begin{aligned}
b= & -\lambda a^{2} \rho / 2 p(\lambda) \\
c= & -a^{3}\left(p(\lambda)+(\lambda-2) \rho-\frac{1}{2} \sigma\right) / p(\lambda) \\
d= & a^{2}(p(\lambda)+(\lambda-2) \rho) / p(\lambda) \\
e= & a^{2} \rho / 2 p(\lambda) \\
4 p(\lambda)^{2} M / a^{4}= & 4 p(\lambda)^{2}-8 p(\lambda) \rho-\left(3 \lambda^{2}-8 \lambda+6\right) \rho^{2}-4 p(\lambda) \sigma \\
16 p(\lambda)^{4} N / a^{8}= & -32 p(\lambda)^{4}-96(\lambda-2) p(\lambda)^{3} \rho-4\left(25 \lambda^{2}-96 \lambda+96\right) p(\lambda)^{2} \rho^{2} \\
& -8\left(5 \lambda^{3}-26 \lambda^{2}+48 \lambda-31\right) p(\lambda) \rho^{3}-\left(2 \lambda^{2}-6 \lambda+5\right)\left(2 \lambda^{2}-2 \lambda-3\right) \rho^{4} \\
& +16[p(\lambda)+(\lambda-2) \rho]^{2} p(\lambda) \sigma .
\end{aligned}
$$

Now (9) represents the equation of an elliptic curve $E$ over the field $\mathbf{Q}(\lambda)$, and any point of $E$ defined over $\mathbf{Q}(\lambda)$ gives rise via the maps (10) to an identity (1). For example, let $A$ be the point of $E$ given by

$$
A=(0,2 p(\lambda))
$$

Then $(M, N)=\left(-a^{4}, 0\right)$ and the associated quartic curve may be taken in the form $X^{4}-Y^{4}=0$. Similarly $-A=(0,-2 p(\lambda))$ gives rise to the quartic curve $X^{4}+3 Y^{4}-4 Z^{4}=$ 0 . These two curves, however, clearly possess points rational over $\mathbf{Q}(\lambda)$ (indeed, over $\mathbf{Q}$ ). But consider instead the point $2 A=\left(-\lambda^{2},-4\right)$ on $E$; this gives rise to the example given as III(a) in Bremner, Lewis, Morton [1]. Further, the point at infinity on $E$ gives rise to the example III(b).

3. If we let $B$ be the point of $E$ given by

$$
B=\left(-\lambda^{2}+1, \lambda^{2}+1\right)
$$


then

$$
A-B=\left(\frac{4\left(\lambda^{3}-2 \lambda^{2}+2\right)}{(\lambda-1)^{2}}, \frac{-2\left(\lambda^{3}-2 \lambda^{2}+2\right)\left(\lambda^{3}+\lambda^{2}-7 \lambda+9\right)}{(\lambda-1)^{3}}\right) .
$$

Denoting this point by $P$, it is easy to verify that $P$ has infinite order in the group of $\mathbf{Q}(\lambda)$-rational points of $E$.

Remarks. It is well-known that the group of $\mathbf{Q}(\lambda)$-points on $E$ is finitely generated; it seems plausible that the rank of the group is 2 with generators $A, B$ at (11), (12), but this has not been specifically checked.

THEOREM. Let $m \in \mathbf{Z}, m \equiv 1 \bmod 9$. Then the point $m P$ of $E$ gives rise in the manner described above to a quartic curve $\Gamma: X^{4}+M Y^{4}+N Z^{4}=0$ which possesses no point defined over $\mathbf{Q}(\lambda)$, but does have a point defined over a cubic extension of $\mathbf{Q}(\lambda)$.

Proof. The method is to localize at the prime ideal $(\lambda)$ of $\mathbf{Q}[\lambda]$, thereby restricting attention to the constant terms of all the polynomials.

Indeed, $P$ specializes to the point

on the curve

$$
P_{0}=(8,36)
$$

$$
E_{0}: S^{2}=R^{3}+16 R^{2}-32 R+16
$$

Considering the further reduction modulo $5, P_{0}$ corresponds to the point

$$
\tilde{P}_{0}=(3,1)
$$

on the curve

$$
\tilde{E}_{0}: s^{2}=r^{3}+r^{2}+3 r+1 .
$$

Now $2 \tilde{P}_{0}=(2,2) ; 3 \tilde{P}_{0}=(0,1) ; 4 \tilde{P}_{0}=(1,4) ; 5 \tilde{P}_{0}=(1,1)$, so that $\tilde{P}_{0}$ is of order 9 on $\tilde{E}_{0}$. It follows that for $k \in \mathbf{Z}$, then $Q_{k}=(9 k+1) P_{0} \equiv P_{0} \equiv(3,1) \bmod 5$. Such points $Q_{k}$ give rise to quartic curves

$$
\Gamma: X^{4}+M Y^{4}+N Z^{4}=0
$$

where from (10), with obvious notation, $M_{0} \equiv a^{4}, N_{0} \equiv a^{8} \bmod 5$.

In particular, taking a non-zero mod 5 , then

$$
M_{0} \equiv N_{0} \equiv 1 \bmod 5 \text {. }
$$

Suppose now $(x, y, z)$ is a point of (15) defined over $\mathbf{Q}(\lambda)$, where $x, y, z$ have no common factor. Then specializing to $\lambda=0$ results in the rational identity

$$
x_{0}^{4}+M_{0} y_{0}^{4}+N_{0} z_{0}^{4}=0
$$

which by (16) forces $x_{0}=y_{0}=z_{0}=0$. Then $x, y, z$ are all divisible by $\lambda$, a contradiction. Thus $\Gamma$ has no non-trivial $\mathbf{Q}(\lambda)$-point.

To show (15) has a point defined over a cubic extension of $\mathbf{Q}(\lambda)$, it suffices to show 
from (1) that the corresponding cubic $x^{3}+a x^{2}+b x+c$ is irreducible over $\mathbf{Q}(\lambda)$. But from (10), specializing to $\lambda=0$,

$$
\left(a_{0}, b_{0}, c_{0}\right) \equiv\left(a_{0}, 0, a_{0}^{3}\right) \bmod 5 .
$$

Now the cubic polynomial $\chi^{3}+a_{0} \chi^{2}+a_{0}^{3}$ is irreducible mod 5 , and so $x^{3}+a x^{2}+b x+c$ is irreducible over $\mathbf{Q}(\lambda)$.

4. Remark. Although (15) is locally insolvable at the prime $(\lambda)$, it is solvable modulo $\mathfrak{p}$ for those prime divisors $\mathfrak{p}$ of $M, N$. For from (2)-(5)

$$
\begin{aligned}
M & =\left(4 e^{3}+c^{2}\right) / d+(4 c-d) d-6 e^{2} \\
N & =-d c^{2}-e^{4} ;
\end{aligned}
$$

then on eliminating $d$ :

$$
\left(c^{2}+e^{3}\right)^{4}+M(c e)^{4} \equiv 0 \bmod N
$$

and on eliminating $c^{2}$ :

$$
(d-e)^{4}+N \cdot 1^{4} \equiv 0 \bmod M .
$$

These lift by Hensel's Lemma to $p$-adic solutions (at least, in the former instance, for $(c e, N)=1)$.

Finding an infinite family of examples where each member is everywhere locally solvable, seems quite a difficult problem.

\section{REFERENCES}

1. A. Bremner, D. J. Lewis and P. Morton, Some varieties with points only in a field extension, Archiv der Math. 43 (1984), 344-350.

2. J. W. S. Cassels, On a problem of Pfister about systems of quadratic forms, Archiv der Math. 33 (1979), 29-32.

3. D. F. Coray, Algebraic points on cubic hypersurfaces, Acta Arith. 30 (1976), 267-296.

4. D. F. Coray, On a problem of Pfister about intersections of three quadrics, Archiv der Math. 34 (1980), 403-411.

5. A. Pfister, Systems of quadratic forms, Colloque sur les formes quadratiques, 2 Bull. Soc. Math. France, Mem. No. 59, (1979), 115-123.

Department of Mathematics

Arizona STate University

TEMPE AZ 85287

U.S.A. 